\title{
RSS Based Bridge Scour Measurement Using Underwater Acoustic Sensor Networks
}

\author{
Prabhat Dahal, Dongming Peng, Yaoqing (Lamar) Yang, Hamid Sharif \\ Department of Computer and Electronics Engineering, University of Nebraska-Lincoln, Lincoln, USA \\ Email: prabhat.dahal@huskers.unl.edu \\ Received July 2013
}

\begin{abstract}
Bridge Scour is one of the major causes of bridge failures all around the world and there have been significant efforts for its detection and measurement using different acoustic approaches. In this paper, we propose and investigate an effective method to utilize Received Signal Strength (RSS) for measuring scour depth where acoustic sensors are deployed. We also extend a statistical testing to determine the difference in signal levels at the sensor nodes prior to and after scour formation and subsequently determine the actual depth of scour. Additionally, we make an attempt to evaluate underwater distance and depth using signal strength perceived at the receiver which makes it free from the requirement of accurate receiver-sender synchronization in contrast to Time of Flight (ToF) or Time of Arrival (ToA) techniques. The scour depths are eventually compared for the conditions when the bottom is composed of a single or multiple layers. The simulation results clearly show that different depths are calculated for the case of multilayered bottom (0.8 to 3.9 meters for instance) as compared to a constant depth of 2 meters for the case of a single layered bottom.
\end{abstract}

Keywords: Bridge Scour; Received Signal Strength; Acoustic Wave Propagation; Underwater Acoustic Sensor Networks

\section{Introduction}

Bridge scour is considered to be one of the main causes of bridge failures all over the world. For instance, tentatively $60 \%$ of more than 1000 bridge collapses in the United States in the past 30 years are linked to the scour of bridge foundations [1]. The problem of such gravity is further aggravated by the fact that the annual cost for scour-related bridge failures only, was estimated to be around $\$ 30$ million, according to a study by the Transportation Research Board in 1997 [2].

The process of formation of a bridge scour can be credited to the erosive action of flowing water. Flowing water tends to excavate and carry with it sediment materials from the water bed and from around the bridge columns, piers and abutments. Formation of bridge scour is a complex and dynamic process that depends on factors such as the water depth, pier and abutment shape and width, the velocity of flow, composition and material properties of the sediments underneath the water body, and many more. Normally, three different types of scours are considered-local scour, contraction scour and degradational scour. Local scour is the type that we are dealing with in this paper, which is the washing away of sediments from around piers and abutments creating scour holes.
Several means have been tried for the measurement of scour depth in the field. Early on, radar and sonar were employed to successfully measure the scour depth. Radar sends out electromagnetic waves to the water bottom, which then reflects off the bottom interface and reaches the transmitter. Similarly, sonar is a technique that uses sound propagation in the same way as a radar does. Sonar is specifically used for underwater purposes. The time taken by the radar and sonar waves to travel from transmitter to the receiver after undergoing reflection off the bottom is used to compute the range and hence the depth.

Despite being successful in measuring scour depth, the use of radar and sonar is limited by the fact that they are usually only used to determine the final stage of the sediment distribution around the pier and there is no continuous monitoring. In recent years, techniques employing Time-Domain Reflectometry (TDR) [3] and Fiber Bragg Grating (FBG) sensors [4] have emerged to facilitate real-time monitoring of bridge scour. It is to be noted that several mechanical systems also exist for the measurement of bridge scour, with one of them being sliding magnetic collar [5]. However, the major issue with this system is that it suffers from the problem of jamming and cannot be reversed for reuse.

Recently, water bottom sensor nodes have been consi- 
dered to extend applications beyond underwater environment monitoring like pollution monitoring, oceanographic data collection and offshore exploration, and used for bridge monitoring and tactical surveillance. In order to achieve this, we need to establish communication between underwater devices. Underwater Acoustic Sensor Networks (Underwater-ASN) consist of a variable number of sensors that are deployed to conduct the task of collaborative monitoring over a particular area of interest. However, there are several hindrances when it comes to the deployment of underwater acoustic sensors. Underwater acoustic communications are mainly influenced by path loss, noise, multi-path, Doppler spread and high and variable propagation delay. All these factors combine to affect the temporal and spatial variability of the underwater acoustic channel and make the available bandwidth of the Underwater Acoustic (UWA) channel limited and dramatically dependent on both range and frequency. Provided that underwater acoustic channel has several complexities as such, long-range systems may have a bandwidth of only a few $\mathrm{KHz}$, while short-range systems may have a more than a hundred $\mathrm{KHz}$ bandwidth.

The rest of the paper is organized as follows: Section II describes how distances or depths can be estimated by Received Signal Strength (RSS) method. Section III provides a detail of the proposed architecture explaining how the sensors are positioned and the signals are received to make estimations. Section IV includes the simulation results that point out the deviation in scour depth from normal when multilayered bottom is taken into account. Finally, Section V presents our conclusions.

\section{RSS Based Range Estimation}

There are different techniques that exist for distance measurement in terrestrial wireless communications such as Time Difference of Arrival (TDoA), Time of Arrival (ToA), Received Signal and Strength Indication (RSSI), and Angle of Arrival (AoA), to name a few. TDoA uses two different transmission-media, e.g. Radio Frequency (RF) and acoustic wave, to estimate the distance between two nodes. This estimation is based on different arrival times due to the difference in propagation speeds through the same medium [6]. But RF is not quite applicable for underwater usage because of its limited propagation in water medium caused by high attenuation [7], and therefore, TDoA is not used in aquatic environment. In contrast, AoA method is dependent on a direct line-of-sight (LOS) path from a transmitter to a receiver. This means that multi-path components of the same signal may appear as signals arriving from different (unwanted) directions and can lead to erroneous results in AoA measurements [8].

ToA is extensively used for range measurement in shortrange Underwater Acoustic Sensor Networks (UASNs) $[9,10]$. As its name refers, it utilizes the time taken by an acoustic wave to travel from the transmitter to the receiver [8]. The time taken for travel is used to calculate the distance according to the underwater sound velocity. It is worth mentioning that for accurate results using ToA, the sender and receiver must be accurately synchronized.

For this work, we attempt to make use of a new distance measurement technique (here, depth) based on Received Signal Strength (RSS) for short range underwater acoustic communications. Unlike ToA, in RSS, wireless devices use the received signal power to measure the distance between the sender and the receiver. Seen this way, synchronization is not a problem as in ToA, but simplifying the distance/transmission-loss relationship can be quite complex. The work in [11] shows that the function to express distance in terms of transmission loss required by RSS for distance calculation uses the Lambert $\mathrm{W}$ function. The distance function is derived after sufficient iteration functions. The authors claim that a very accurate calculation of distance can be made in less than four iterations. We utilize the RSS based range estimation approach as proposed by [11] for our research.

Sound loss in water is classified as spreading loss and attenuation loss. Spreading loss includes spherical 1) and cylindrical 2) losses, which is caused by the expansion of an acoustic wave as it propagates through a medium, and attenuation includes absorption, scattering and diffraction [12]. Accurate values for attenuation can be measured by considering the effects of transmission medium (salinity, pressure, temperature and many others) and environment parameters like air bubbles within water volume, absorption by the sediments, reflection off the surfaces and scattering. Here, only transmission medium parameters are considered for the ease of calculation. Transmission losses can be mathematically expressed as

$$
\begin{aligned}
T L s p h & =20 \log (\text { Dist }) \\
\text { TLcyl } & =10 \log (\text { Dist })
\end{aligned}
$$

where the first equation gives an expression for the transmission loss with respect to spherical propagation of the wave and the second equation is that with respect to cylindrical propagation. So the general expression for transmission loss in sea water [12] is given by

$$
\text { TLtotal }=T L s p h+T L c y l+10^{-3} \alpha \text { Dist }
$$

where $\alpha$ is the absorption coefficient in sea water as expressed in (4), given by the Thorp's absorption coefficient [13], which depends only on frequency $\mathrm{f}$ below 50 $\mathrm{kHz}$,

$$
\alpha=1.0936\left[\frac{0.1 f^{2}}{1+f^{2}}+\frac{40 f^{2}}{4100+f^{2}}\right]
$$

Here, the factor 1.0936 is used to change the unit of the coefficient from $\mathrm{dB} / \mathrm{kyd}$ to $\mathrm{dB} / \mathrm{km}$. The authors in [11] 
consider spherical spreading to be good enough to model a wide range of available data. Hence, (3) reduces to a total transmission loss given by

$$
\text { TLtotal }=20 \log (\text { Dist })+10^{-3} \alpha \text { Dist }
$$

Equation (5) means that the total transmission loss can be expressed in terms of the path loss due to spherical spreading and absorption. Now, the distance can be calculated using the following relation as expressed in [11],

$$
\text { Dist }=\frac{20000 \times W\left(\frac{\ln (10)}{20000} \alpha e^{\lambda T L}\right)}{\alpha \ln (10)}
$$

where $W$ is the Lambert function.

Given that we have $A_{1}=10000 /(\lambda \alpha), A_{2}=1 / A_{1}$ and $A_{3}$ $=\lambda T L$, we can write,

$$
\text { Dist }=A_{1} \times W\left(A_{2} \times e^{A_{3}}\right)
$$

where Dist refers to the distance from the sensor to the point of reflection and back. The scour depth that we seek to measure will be half the distance measured.

\section{Communication Architecture}

Underwater Wireless Acoustic Sensor Networks (Underwater-ASN) are equipped with a number of acoustic sensors that work collectively to achieve the monitoring requirement imposed upon the network. The deployment of the sensors depends on various dynamic underwater environments. However, the overall Underwater-ASN can be broadly categorized into two basic communication architectures: two dimensional architecture, where the nodes are attached near to the bottom, and three dimensional architecture, where the nodes float at different depths inside the water body [14].

\subsection{Architecture for Depth Measurement}

Despite the fact that there are three dimensional and two dimensional architectures for underwater-ASN, the former is generally used to provide a complete sampling of the 3D underwater environment, whereas for underwater depth measurements, the latter is preferred. In a three dimensional architecture, the sensors float at different depths within the water volume by means of floating buoys using wires or winches. In contrast, the two dimensional architecture is the one in which the sensor nodes are organized in a cluster fashion and remain statically anchored at or near the water bottom as shown in Figure 1.

The sensor nodes make use of acoustic means to communicate with each other. A central hub exists for each cluster of the sensor nodes and is known as the underwater gateways (underwater-gateways). These hubs act as means to transfer the data collected from the anchored nodes to the surface station and to achieve this, they use a set of vertical and horizontal transceivers. While the horizontal transceivers are used for establishing communication with the sensor nodes to collect monitored data and send some commands, the vertical ones are used to relay the collected data to the surface station. Since the surface station is required to handle multiple communications with the underwater-gateways, it is equipped with a transceiver designed specifically for this purpose. In addition, it also has a long range radio transmitter for the final transmission of collected data to the onshore sink.

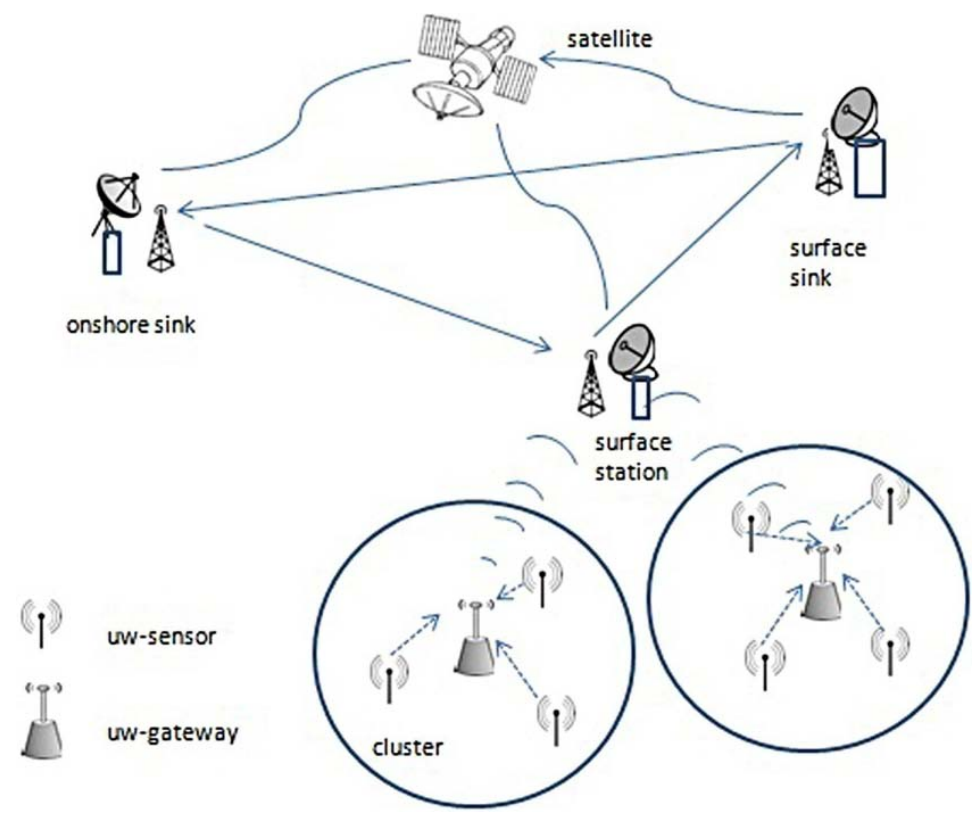

Figure 1. Two dimensional underwater-ASN Architecture. 


\subsection{Proposed Architecture}

The architecture proposed in this paper for the case of bridge-pier scour depth measurement is essentially a two dimensional static architecture as shown in Figure 2. A number of sensor nodes are fixed in each bridge pier near to the water bottom. The nodes in each pier form a cluster and have their own underwater-gateway. The nodes are oriented to direct acoustic waves to the bottom and receive the reflected waves. The collected data (signal strength in this case) is sent through acoustic links to the gateway located in the same pier as the sensor nodes. The underwater-gateway sends the data to the surface station (which can be located in the bridge structure near the water surface) for final relay to the onshore sink.

\subsection{Signal Analysis}

The underwater acoustic channel is complex, exhibiting different behaviors under different conditions. The speed of sound that varies with depth causes propagation delays. In addition, scattering, multipath interference are examples of phenomena that make the UWA channel complex. Different research suggests different ideas. Rayleigh fading was assumed in [15]. As research in UWA progressed, it was proposed that several distinct paths called eigenpaths exist in an UWA channel [16]. The signals exist over all these paths. Moreover, each path contains sub paths called eigen-rays, comprising of a dominant path and other smaller paths. The number of eigen-rays reach- ing the receiver is a Poisson's distribution. Saleh-Valenzula model [17] has been proposed for UWA networks in [18]. According to S-V model, usually, multipath signals of the same pulse arrive at the receiver in clusters and two Poisson models are employed to model the path arrivals, one for the first path of different clusters and the other for the rays within each cluster. In an Ultra-Wide Band-SV (UWB-SV) model, the $k$-th path within the $l$-th cluster is denoted by $\alpha_{k l}(u)$ whose magnitude, $\left|\alpha_{k l}(u)\right|$, follows a Rayleigh distribution.

According to [18], the reflected signal from the bottom received by sensor $m$ is given by

$$
Z_{m}(u) \approx \gamma \sum_{l} \sum_{k} \alpha_{k l}(u)+n(u)
$$

which is received by summing all the received signals in a pulse duration and $\gamma$ is the received signal strength.

For $M$ sensor nodes, $\boldsymbol{y}=\left[y_{1}, y_{2}, y_{3}, \ldots, y_{M}\right]$, then,

$$
f(y)=\prod_{m=1}^{M} f\left(y_{m}\right)
$$

and the unique solution of the maximum- likelihood estimate of the signal poweris given by

$$
\hat{\theta}_{M L}=\frac{\sum_{m=1}^{M} y_{m}^{2}}{2 M}-\sigma^{2}
$$

where, $y_{m}=\left|Z_{m}(u)\right|$ follows Rayleigh distribution, $\sigma^{2}$ is the variance of noise $n(u)$ with zero mean complex Gaussian distribution and $M$ is the number of sensor nodes.

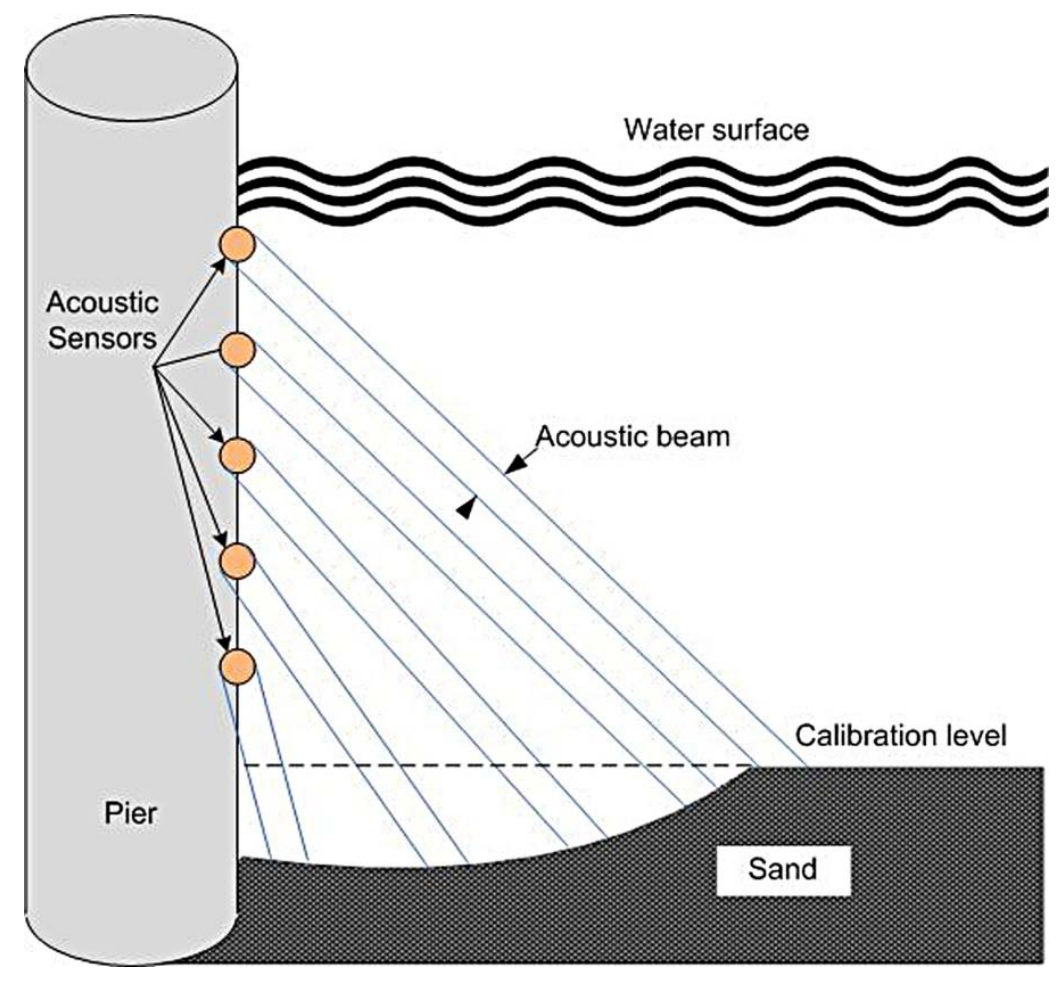

Figure 2. Proposed underwater-ASN architecture. 
Let us define the estimates of received signal power $(\theta$ $\left.=\gamma^{2} / 2\right)$ before and after scour formation as

$$
\hat{\theta}_{M L 1}\left(y_{m 1}\right) \text { and } \theta_{M L 1}\left(y_{m 1}\right)
$$

The estimates are gamma distributed (parameter $k$ and $\theta$ ). For sufficiently large number of sensor nodes, the estimates tend to normal distribution with mean, $\mu=k \theta$ and variance $=k \theta^{2}$. Then,

$$
\hat{\theta}_{M L 1}=\frac{\sum_{m=1}^{M} y_{m 1}^{2}}{2 M}-\sigma^{2}
$$

and

$$
\hat{\theta}_{M L 2}=\frac{\sum_{m=1}^{M} y_{m 2}^{2}}{2 M}-\sigma^{2}
$$

Given that a scour has been formed, the signal incident at the bottom now has to undergo an additional path through water and be reflected off the bottom comprising of a dense layer of water and a solid half space. It is obvious that the signal reaching the sensor in this case weakens in strength, owing to the additional transmission loss and the reflection loss. We seek to calculate the difference in the estimate at the receiver and difference in mean of the two estimates is employed for this. In order to take into account, the effect of the entire distribution of the estimates, a 95\% Confidence Interval (C.I.) is created that will provide us with a range $[a, b]$, as given in (15) and (16) between which the difference of mean of the estimates is likely to exist. We have proven this through the simulations that the higher the number of sensors, the more accurate the confidence interval is. For a 95\% significance level (two tailed), the C.I. is given by,

$$
\text { C.I. }=\left(\mu_{1}-\mu_{2}\right) \pm 1.96 \sigma_{\theta 1-\theta 2}
$$

such that,

$$
a=\left(\mu_{1}-\mu_{2}\right)-1.96 \sigma_{\theta 1-\theta 2}
$$

and

$$
b=\left(\mu_{1}-\mu_{2}\right)+1.96 \sigma_{\theta 1-\theta 2}
$$

where $a$ and $b$ are the lower and upper limits of the confidence interval respectively and 1.96 is the z-value from the z-table corresponding to the $95 \%$ significance level.

If $a$ and $b$ are both positive in C.I. $=[a, b]$, we are 95\% confident that there has been a decrement in received signal power. Assuming similar conditions of water and the environment, the signal has probably travelled additional path (depth) and there could be a probable scour formation which can be checked for.

The difference in signal power $\left(\gamma^{2} / 2\right)$ thus estimated is due to the additional Transmission Loss and Reflection Loss (RL). From Figure 3(a), for the condition prior to scour formation, we have,

$$
S L 1=S L 0-T L 1-R L 1
$$

where $S L 1=20 \log \left(\gamma_{1}^{2}\right)$ is the received signal level at the sensors.

Similarly, after the scour has been formed, as shown in Figure 3(c), we have the expression for received signal level at the sensors as,

$$
S L 2=S L 0-T L 1-R L 1-T L 2-R L 2
$$

where $S L 2=20 \log \left(\gamma_{2}^{2}\right)$ is the received signal level, $T L 2$ is the transmission loss in water with depth $d_{0}$ and $R L 2$ is the reflection loss from the layered bottom, with the depth of sediment layer being $d_{1}$.

From (17) and (18), we have,

$$
20 \log \left(\frac{\gamma_{1}^{2}}{\gamma_{2}^{2}}\right)=R L 2+T L 2-R L 1
$$

where $R L 2$ is the reflection loss from the layered bottom expressed as $R L 2=-20 \log \left(R_{\mathrm{b}}\right)$.

Provided that we make an estimate of $\gamma_{1}$ and $\gamma_{2}$, we can calculate $R L 1, T L 2$ for a particular depth $d_{1}$ and this would lead us to estimate the depth $\mathrm{d}_{0}$ using (7). The total depth would then be $D=d_{0}+d_{1}$. Despite the fact that $d_{1}$ is the depth of the sediment layer, it should be noted that this layer is unconsolidated, which is considered liquid and would not provide support required for the pier.

In contrast, when the bottom after scour formation is considered to be a single layered half space, (19) becomes

$$
20 \log \left(\frac{\gamma_{1}^{2}}{\gamma_{2}^{2}}\right)=T L 2
$$

with $T L 2$ being accountable for the entire depth $D=d_{0}$ and $d_{1}=0$.

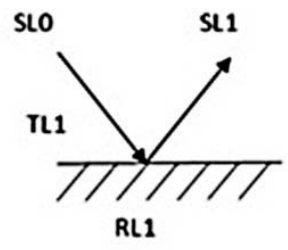

(s)

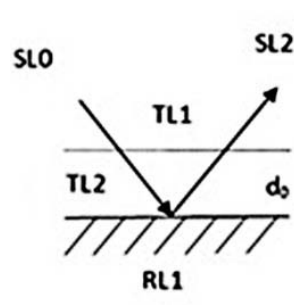

(b)

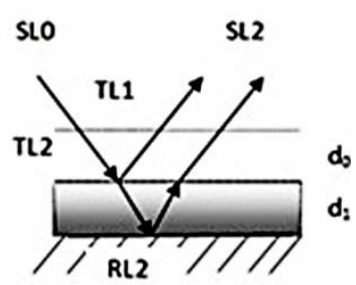

(c)
Figure 3. Reflection and Transmission Losses when (a) no scour has been formed (b) the bottom is a single layer halfspace and (c) the bottom is composed of unconsolidated sediment layer over solid half-space. 


\section{Simulation Results}

The simulation results presented in this section are based on assumed values as given in Table $\mathbf{1}$ and were implemented in MATLAB on a Intel(R) Core(TM)2Duo 2.00 $\mathrm{GHz}$ processor. The frequency of acoustic wave chosen is $10 \mathrm{KHz}$ which gives a Thorp's absorption coefficient, $\alpha$, equal to 1.1498 .

For a significance level of 95\%, we have the Confidence Interval (C.I.) as

$$
\text { C.I. }=\left(\mu_{1}-\mu_{2}\right) \pm 1.96 \sigma_{\theta 1-\theta 2}
$$

where, the z-value 1.96 corresponds to $95 \%$ significance level from the z-table.

Different values of received signal strengths at the sensor nodes are assumed for two different conditions- the initial one, where the acoustic signals reflect off the solid half layered bottom, which is taken as a reference; and the second one, where a scour has been formed and the bottom has shifted to a certain depth from the reference level. For the second case, as per our assumption, the bottom consists of a dense liquid layer atop a solid half layered bottom, thereby causing the reflected signals reaching the sensors to be of lesser strength than in the initial condition.

The graphs in Figure 4 present different instances of calculated C.I. that indicate that we are 95\% confident of the difference in signal powers between the initial and the latter condition being in between the lower limit (the bar on the left) and the upper limit (the bar on the right), for the case of 50 sensor nodes. The line between the upper limit and the lower limit in each pair of bars indicate the actual difference in signal levels.

Talbe 1. Assumed Data For Water Bottom Layers.

\begin{tabular}{ccccc}
\hline Layer & Thickness & $\boldsymbol{c}_{\boldsymbol{p}}(\mathbf{m} / \mathbf{s})^{\mathbf{a}}$ & $\boldsymbol{c}_{\boldsymbol{s}}(\mathbf{m} / \mathbf{s})^{\mathbf{b}}$ & $\boldsymbol{\rho}\left(\mathbf{g} / \mathbf{c m}^{3}\right)$ \\
\hline 1 & 1 & 1700 & 0 & 1.8 \\
2 & $\infty$ & 4700 & 1900 & 2.5 \\
\hline
\end{tabular}

${ }^{\mathrm{a}}$ Compression wave velocity; ${ }^{\mathrm{b}}$ Shear wave velocity.

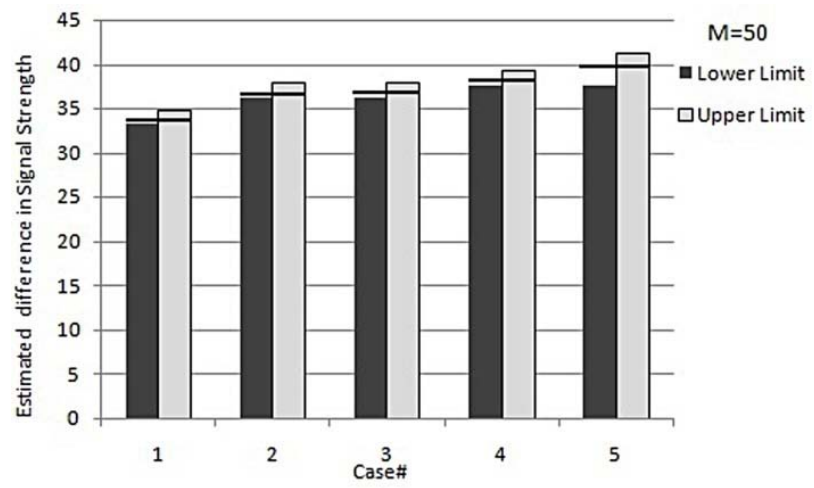

Figure 4. Confidence Interval for difference in signal strength at the receiver $(M=50)$.
Figure 5 presents similar graphs for the cases of the number of nodes being 5 and 80 respectively. It can clearly be seen that, as the number nodes increases, the $95 \%$ C.I. of the estimate of difference in signal powers narrows and gets closer to the actual value. The result is the opposite when the number of sensor nodes is decreased. This shows that, for our C.I. to be a more accurate representation of difference in signal powers, a greater number of sensor nodes is preferred.

The second section of the simulation, as shown in Figure 6, comprises of the part where the effect of layered bottom is compared to non-layered bottom with respect to scour-depth measurement. For different data, as given in Table 1, scour depths are measured for the condition when the bottom is composed of a single layer, and the condition when the bottom is composed of two layers (a sediment layer of a certain thickness $d_{1}$, treated as liquid and a solid bottom). The graphs present the results for the signal powers in case 1 of Figure 5, for the case of 80 sensor nodes.

Figure 6 expresses that the total scour depth, for the same received signal strength, depends on the thickness of the sediment layer. The bars represent the total depth (including the additional water depth, $d_{0}$, and the sediment layer depth, $\left.d_{1}\right)$. For each depth, different thickness

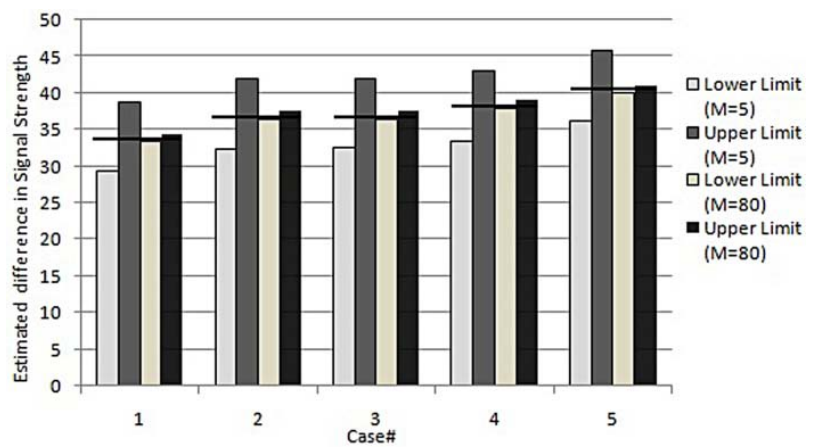

Figure 5. Confidence Interval for difference in signal strength at the receiver $(M=5, M=80)$.

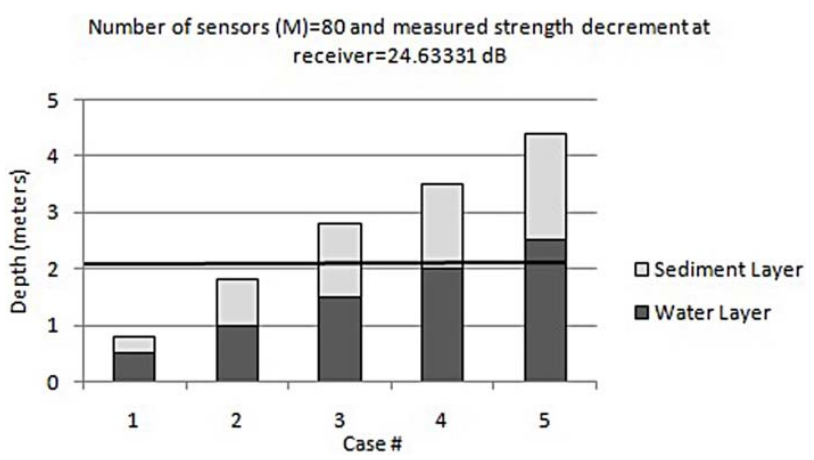

Figure 6. Comparison of scour depth with and without layered bottom. Bars for the layered bottom show the variation in total depth with the depth of the sediment layer. 
of the sediment layer is considered and the water depth is calculated to account for the total loss in signal strength as compared to the reference level. As the sediment layer depth increases (shown by the lower portion of the bars), the corresponding water layer depth also increases, thus increasing the total scour depth. For instance, for a 24.63 $\mathrm{dB}$ signal loss measured at the receiver (with respect to the condition when no scour was formed), an assumption of 0.5 meters sediment layer thickness leads to a total scour depth of approximately 0.8 meters. Likewise, for the same signal loss measured, assuming a sediment layer depth of 2 meters gives a total scour depth of around 1.8 meters.

However, the scour depth without considering the layered approach (when the bottom is a single layered half space), as shown by the straight line in Figure 6, is constant and seems to deviate from the depths calculated for a layered bottom. This shows that the single layered approach, which is inconsistent with the real underwater scenario as pointed out in literature, gives misleading values for scour depth and the effect of sediment layer thickness on scour depth cannot be neglected. The formation of dense sediment layer above solid water bottom results in different values of scour depth.

\section{Conclusion}

Scour holes that tend to make bridge foundations weaker to collapses should be accurately measured. We have successfully shown the RSS for acoustic sensor networks. Unlike ToF or ToA methods for range measurement in underwater environment, in this paper we provided RSS approach to measuring bridge scour depth. Simulations show that the estimation of scour depth tends to approach the real depth as the number of acoustic sensors employed is increased. Also, since in real underwater conditions, erosion of sediments leads to the formation of a dense water layer over the water bottom due to the dissolved sediments, an effort is made to compare the effect of layered nature of water bottom on scour depth to that of non-layered approach- the layered approach giving more accurate values for scour depth. For instance, as seen in the simulations, a sediment layer depth of 1.5 meters results in a total scour depth of 2.8 meters and this value increases with the thickness of the sediment layer. In contrast, when the single layered bottom is considered, the resultant scour depth is about 2 meters. However, since a single layered bottom assumption is fairly unrealistic, the result obtained could be misleading.

\section{Acknowledgments}

This work was sponsored in part by an Interdisciplinary Research Grant of the University of Nebraska-Lincoln.

\section{REFERENCES}

[1] A. M. Shirhole and R. C. Holt, "Planning for a Comprehensive Bridge Safety Program,” Transportation Research Record No. 1290, Transportation Research Board, National Research Council, Washington, D.C., 1991.

[2] P. F. Lagasse, E. V. Richardson, J. D. Schall and G. R. Price, "Instrumentation for Measuring Scour at Bridge Piers and Abutments," National Cooperative Highway Research Program (NCHRP) Paper No. 396, Transportation Research Board, National Research Council, Washington, D.C., 1997.

[3] N. E. Yankielun and L. Zabilansky, "Laboratory Investigation of Time-Domain Reflectometry System for Monitoring Bridge Scour,” Journal of Hydraulic Engineering, Vol. 125, No. 12, 1999, pp. 1279-1284. http://dx.doi.org/10.1061/(ASCE)0733-9429(1999)125:12 (1279)

[4] Y. B. Lin, J.-C. Chen, K.-C. Chang, J.-C. Chern and J.-S. Lai, "Real Time Monitoring of Bridge Scour Using Fiber Bragg Grating Sensors,” Smart Materials and Structures, Vol. 14, No. 4, 2005, pp. 664-670. http://dx.doi.org/10.1088/0964-1726/14/4/025

[5] J. -Y. Lu, J. -H. Hong, C. -C. Su, C. -Y. Wang and J. -S. Lai, "Field Measurements and Simulation of Bridge Scour Depth Variation During Floods,” Journal of Hydraulic Engineering, Vol. 134, No. 6, 2008, pp. 810-821. http://dx.doi.org/10.1061/(ASCE)0733-9429(2008)134:6( $\underline{810)}$

[6] N. B. Priyantha, A. Chakraborty and H. Balakrishnan, "Cricket Location Support System," Proceedings of the Annual International Conference on Mobile Computing and Networking, MOBICOM, 2000, pp. 32-43.

[7] J. Heidemann, W. Ye, J. Wills, A. Syed and Y. Li, "Research Challenges and Applications for Underwater Sensor Networking," IEEE Wireless Communications and Networking Conference, WCNC, Vol. 1, 2006, pp. 228235.

[8] G. Mao, B. Fidan and B. D. Anderson, "Wireless Sensor Network Localization Techniques,” Computer Networks, Vol. 51, No. 10, 2007, pp. 2529-2553. http://dx.doi.org/10.1016/j.comnet.2006.11.018

[9] M. Erol, L. Vieira, A. Caruso, F. Paparella, M. Gerla and S. Oktug, "Multi Stage Underwater Sensor Localization Using Mobile Beacons," Second International Conference on Sensor Technologies and Applications, 2008. SENSORCOMM '08, 2008, pp. 710-714.

[10] E. Kim, S. Woo, C. Kim and K. Kim, "Lamsm: Localization Algorithm with Merging Segmented Maps for Underwater Sensor Networks," Lecture Notes in Computer Science (including subseries Lecture Notes in Artificial Intelligence and Lecture Notes in Bioinformatics), Vol. 4809 NCS, 2007, pp. 445-454.

[11] M. Hosseini, H. Chizari, C. K. Soon and R. Budioarto, "RSS-Based Distance Measurement in Underwater Acoustic Sensor Networks: An Application of the Lambert W Function," Proceedings of the 4th International Conference on Signal Processing and Communication Systems, ICSPS, Gold Coast, 2010, pp. 1-4.

[12] P. Etter, "Underwater Acoustic Modeling and Simulation," 
3rd Edition, Spon Press, 2003. http://dx.doi.org/10.4324/9780203417652

[13] W. H. Thorp, "Analytic Description of the Low-Frequency Attenuation Coffcient, "Acoustical Society of America Journal, Vol. 42, 1967, p. 270. http://dx.doi.org/10.1121/1.1910566

[14] I. F. Akyildiz, D. Pompili, T. Melodia, "Challenges for Efficient Communication in Underwater Acoustic Sensor Networks," ACM SIGBED Review-Special Issue on Embedded Sensor Networks, ACM, NY, 2004, pp. 3-8.

[15] J. A. Catipovic, A. B. Baggeroer, K. Von Der Heydt and D. Koelsch, "Design and Performance Analysis of a Digital Telemetry System for Short Range Underwater Channel," IEEE Journal of Oceanic Engineering, Vol. OE-9, No. 4, 1984, pp. 242-252.
http://dx.doi.org/10.1109/JOE.1984.1145632

[16] X. Geng and A. Zielinski, "An Eigenpath Underwater Acoustic Communication Channel Model,” OCEANS '95. MTS/IEEE, Challenges of Our Changing Global Environment, Conference Proceedings, Vol. 2, 1995, pp. 1189-1196.

[17] A. A. Saleh and R. A. Valenzuela, "A Statistical Model for Indoor Multipath Propagation,” IEEE Journal on Selected Areas in Communications, Vol. 5, No. 2, 1987, pp. 128-137.

[18] Q. Liang and X. Cheng, "Underwater Acoustic Sensor Networks: Target Size Detection and Performance Analysis," Proceedings of IEEE International Conference on Communications, Beijing, May 2008, pp. 3151-3155. 